\title{
SOCIEDAD ACTUAL E INMIGRACIÓN: EL RETO DE LA ENFERMERÍA HUMANISTA
}

\section{Ruiz Salvador, D; Torralbo O jeda, E; O rtiz Morales, M.A; del Pino Alcaraz, M.I.; Artero López, C.}

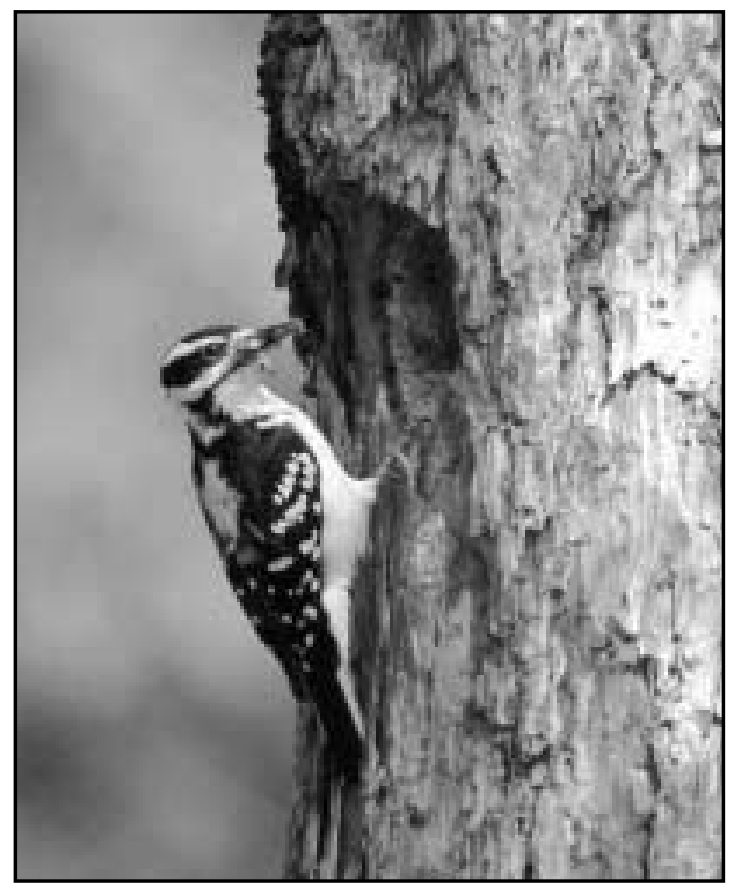

NOWADAYS SOCIETY AND IMMIGRATION: A CHALLENGE FOR HUMANISTIC NURSING

\section{INTRODUCCIÓN Y OBJETIVOS}

La realidad demográfica de nuestro planeta hoy por hoy, está abocada a la multiculturalidad. Así muchos conceptos como el de salud y enfermedad deben ser redefinidos en función a la nueva situación social así como las relaciones a establecer entre personal sanitario-usuario del sistema. La enfermería, por su formación orientada a la persona como un conjunto bio-psico-social y cultural, es la profesión que más puede acercarse a la respuesta que poco a poco se va solicitando del mundo asistencial. El abordaje de campos de actuación que quedaron en segundo plano mientras el sistema sanitario concebía la atención desde una perspectiva biologicista, vuelven a cobrar sentido ahora y de forma progresiva en la medida en que la sanidad se hace más permeable a una concepción de la persona más humanista.

La salud deviene como un fenómeno estrechamente ligado a las condiciones de vida de la población, que sólo puede ser explicado por medio de un enfoque integral y sistémico. Es un proceso inmerso en la dinámica social donde se pueden identificar seis grandes dimensiones: biológica, ecológica, sociológica, psicológica, económica y de los servicios de salud. Las ciencias sociales han realizado y hacen grandes aportes a la nueva concepción o visión que proponen los estudiosos de cómo influyen los problemas sociales en la salud humana, a la hora de abordar el proceso salud enfermedad, y pretenden no sólo identificar en el hombre la enfermedad que lo aqueja, la biología de esta, su causa y la conducta a seguir; sino verlo y abordarlo como un ser no sólo biológico sino también psicosocial que siente, sufre y padece, o sea, donde la espiritualidad también adquiere valor, que reconoce que el hombre vive inserto en sociedad o en un entorno determinado, por tanto, todas las relaciones que establezca dentro de éste, de alguna manera, influyen positiva o negativamente sobre él, puesto que el hombre es un producto de su medio.

También la teoría de los cuidados culturales de Madeleine Leininger trata sobre la importancia de brindar un cuidado de enfermería según sea la cultura del paciente al que se le esté brindado el cuidado. Leininger desarrollo su teoría de la diversidad y universalidad de los cuidados culturales, basada en su convicción de que las personas de culturas diferentes pueden ofrecer información y orientar a los profesionales para recibir la clase de cuidados que desean o necesitan de los demás. La cultura determina los patrones y estilos de vida que tienen influencia en las decisiones de las personas, esta teoría ayuda a la enfermera a descubrir y documentar el mundo del paciente y utiliza sus puntos de vista émicos, sus conocimientos y sus prácticas 
en conjunción con una ética apropiada (conocimiento profesional) como base para adoptar acciones y decisiones profesionales coherentes con los modos culturales.

Así el papel de la enfermera incrementa su relevancia en las nuevas concepciones sanitarias que giran en torno al individuo como eje del sistema sanitario al ofrecérsele la oportunidad de ajustar su actuación a las pautas culturales, sociales, demográficas, psicológicas, derivadas de las condiciones de vida ... que perfilan las necesidades de los grupos cada vez más heterogéneos.. Es por ello que el profesional de la enfermería debe nutrirse ahora más que nunca del saber de diferentes disciplinas como la antropología, psicología social, sociología o geografía humana, en definitiva, se precisa de un bagaje de conocimientos suplementarios en los que generalizamos como humanidades (o ciencias humanas y sociales) que permitan un abordaje holístico de las necesidades de los individuos y comunidades.

Este es el planteamiento que aceptamos como más acertado en la sociedad de hoy, y en concreto, con respecto a los cuidados en la población inmigrada. Así nos surge la necesidad de llevar a cabo un estudio de los diferentes aspectos referentes a la inmigración, que desde una perspectiva humanística, nos aportan información sobre las pautas de actuación más adecuadas en estos grupos poblacionales. Por tanto nos marcamos como punto de interés de nuestro trabajo el análisis, partiendo de lo general a lo más específico de nuestro ámbito de actuación geográfico, de diferentes elementos demográficos, psicológicos, antropológicos y sociales que marcan unas pautas de actuación muy definidas con respecto a la demanda de asistencia sanitaria en la población inmigrada.

\section{MATERIAL Y MÉTODO}

La determinación de un objetivo a estudio tan amplio obliga necesariamente a la combinación de diferentes métodos para la recopilación y análisis de datos entrelazando tanto técnicas cuantitativas como cualitativas. En este caso el planteamiento es a través de un estudio descriptivo trasversal basado en revisiones bibliográficas, consulta de documentación clínica y humanística, así como aportaciones cualitativas a través de grupos nominales y trabajo de campo elaborados en el último semestre del año dos mil cinco.

\section{RESULTADOS}

Es un hecho evidente que el proceso inmigratorio se ha ido acentuando de manera progresiva en los últimos años, así nos lo demuestran los datos que expone el Instituto Nacional de Estadística (INE) (Tabla 1). Aquí apreciamos el progresivo incremento de individuos que se registran en los padrones municipales de todos los ayuntamientos de España que aparecen nacionalidad no española y lugar de residencia habitual aquí. Hemos evolucionado hacia un crecimiento exponencial si bien primera vista observamos ciertas irregularidades que tienen que ver con los cambios en cuanto a los requerimientos para ser incluidos en el padrón municipal y la influencia de los procesos de regularización, en tal inclusión. En lo referente a la distribución por edad es evidente una franja dominante de edad que abarca entre los 20 a 40 años lo que nos revela que efectivamente hablamos de movimientos demográficos que presentan un clara base laboral con una distribución en cuanto al sexo más o menos equitativa (Tabla 2).

En lo referente a los lugares de destino, vemos que se produce una distribución muy irregular entre las diferentes comunidades, ello nos lleva a pensar que efectivamente aparecen una serie de espacios diana hacia los cuales parece dirigirse en su mayoría la inmigración, estableciendo como límite inferior la mitad del valor porcentual de la comunidad que más inmigrantes recibe (por presentar el resto de comunidades valores poco significativos respecto a las anteriores), observamos que es en Cataluña, Madrid, Comunidad Valenciana y Andalucía donde se concentran casi el $70 \%$ de la población inmigrada (Gráfico 1). También comprobamos cómo Andalucía se encuentra entre aquellas de mayor presencia de inmigrados, por lo que procederemos ahora a desglosar esta distribución por provincias (Tabla 3). De ello obtenemos que la provincia que soporta el mayor número de inmigrados es Málaga seguida de Almería con una diferencia importante con respecto al resto de provincias, pero tratando de establecer la proporción de inmigrados por habitante obtenemos que Almería a pesar de que no es la 
provincia con mayor número de éstos si presenta la mayor proporción de inmigrados empadronados respecto a la población total; con lo cual la sensación subjetiva de confluencia de individuos de diferentes nacionalidades se objetiviza (Tabla 4).

Circunscribiéndonos ahora a nuestro ámbito de actuación, hemos de tener en cuenta que el municipio de Níjar es el que más ha crecido poblacionalmente de todo el levante almeriense en los últimos años, con una sucesiva masculinización de la población que supone un $59 \%$ del total. Ello es debido en gran parte a la afluencia de inmigrados puesto que la estructura de género de este grupo no es igualitaria de tal modo que del total de éstos el $44 \%$ son hombres mientras que de las mujeres empadronadas son sólo un $24 \%$.

En cuanto a la distribución por lugar de procedencia, del total de extranjeros empadronados (9.510 un 39\% de la población), el 59\% son magrebíes, el 24\% de Europa Empobrecida, el 6\% procedentes de la Europa Enriquecida, el 5\% de Latinoamérica, el 4\% sudsaharianos, algo más del $1 \%$ de Asia y menos del 1\% de América del Norte. En los núcleos de más de 1000 habitantes la proporción de población extrajera son: Campohermoso 27\%; San Isidro $16 \%$ y Níjar $2,9 \%$, sin embargo hemos de tener en cuenta que el lugar de residencia de los inmigrados no suele ser en el mismo núcleo urbano, de hecho sólo lo hacen aquellos procedentes de países con rentas iguales o superiores a las españolas. Normalmente la residencia se fija en cortijadas o en las instalaciones de la propia explotación agraria, pues es el sector primario la principal fuente de riqueza de la comarca, aunque la progresiva saturación de la oferta de mano de obra ha ido trasformando esta zona de un lugar de destino a una zona de tránsito hacia destinos con más demanda de trabajadores poco especializados. Si nos centramos en el análisis de estas cortijadas obtenemos una serie de indicadores sobre la calidad de vida que aquí desarrollan los inmigrados (datos obtenidos a través de trabajo de campo y publicados previamente por la Asociación Pro derechos Humanos de Andalucía). En estas cortijadas (13 en toda la comarca) habitan el 54, $1 \%$ de la población inmigrada, y prácticamente el 40-45\% restante en las explotaciones agrícolas. Respecto a la distribución por lugar de origen de los residentes en ellas encontramos: magrebíes $67,4 \%$, sudsaharianos $32,3 \%$ y residentes de países del este $0,3 \%$. La distribución por sexo y edad: hasta un $95,4 \%$ son varones y en los intervalos por edad encontramos que efectivamente un mayor número de individuos tiene entre 20 y 40 años, al igual que la media nacional.

Haremos un inciso para aportar un dato relevante a tener en cuenta; que gran parte de la población inmigrada no consta en el padrón municipal, es más, según datos de la Asociación Pro derechos Humanos de Andalucía, de los inmigrados que residen en los diseminados poblacionales, sólo el 29,5\% están empadronados. Teniendo en cuenta que estos suponen la mayor parte del total de inmigrados, posiblemente nos estemos refiriendo a un grupo de personas que casi triplique los datos con los que trabajamos.

Pasamos ahora al estudio de la situación higiénico sanitaria del lugar de residencia de este grupo de inmigrados, siempre teniendo en cuenta que en las cortijadas es ventajosa respecto a los enclaves de las explotaciones:

1.- Presencia de basuras en los alrededores de las viviendas: 6 cortijadas presentan basuras de manera generalizada, 5 en algunos puntos aislados y en 2 de forma esporádica. A pesar de la instalación de equipamiento para depositar basura recientemente.

2.- Existencia de agua potable corriente: En 8 de las cortijadas no existe este servicio y en las cinco restantes de forma comunitaria.

3.- Corriente eléctrica: 9 enclaves sin posibilidad de acceso a la red y 4 con servicio de forma continua.

En cuanto a la distribución por sexo de los habitantes de las viviendas: en el $80,7 \%$ de los casos en las viviendas sólo habitan hombres, en el 9\% de los casos en el núcleo hay sólo una mujer y el 10,3\% presenta un núcleo familiar completo con hijos. Nos llama la atención el hecho de que los grupos disfrutan de las mejores situaciones de vivienda son normalmente los que cuentan además con alguna mujer que normalmente ha llegado a la zona por reagrupamiento familiar lo que implica que sus familiares o parejas han tenido la posibilidad de mejorar sus condiciones de vida y acceder a la regularización documental. 
Si tratamos ahora los datos relativos a la asistencia sanitaria, obtendremos que el Hospital de Alta Resolución El Toyo abarca un área de influencia en la que habitan alrededor de 55.000 habitantes (54.485 según padrón municipal del 2005), de ellos, algo menos de la mitad (24.435), corresponden al municipio que hemos descrito; las otras dos entidades administrativas que tienen este hospital por centro sanitario especializado de referencia son Almería Periferia y Carboneras, pero es en la primera donde se concentran la mayoría de las explotaciones agropecuarias y por tanto, presenta con diferencia, la mayor tasa de población inmigrada tanto empadronada como no. El servicio de urgencias se convierte en el punto de contacto principal de este grupo con la asistencia sanitaria, tal y como analizaremos. En el periodo comprendido entre julio y diciembre del dos mil cinco (ambos meses inclusive), se atendieron en este servicio 15823 urgencias, de ellas el 52,5\% (8307) correspondían a población adscrita a este centro. El total de asistencias a inmigrados residentes fue de $762(9,2 \%)$, la edad media de estos 30,3 años con una desviación estándar de 12,2, y una proporción de 52,13 varones por cada cien. Referente al lugar de origen, el $49 \%$ eran de origen magrebíes o sudsahariano con una relación por sexos de 94 a $6 \%$ hombre mujer, lo que nos hace caer en la cuenta que la proporción de mujeres en el otro grupo de inmigrados compuesto por nacidos en países del este de Europa y Latinoamérica es similar a la de los hombres en los africanos.

Los motivos de consulta son:

1. Osteomusculares: $17 \%$

2. Traumatismos: $11,7 \%$

3. Dolor abdominal: $13,8 \%$

4. Oticos: $4,3 \%$

5. Odontalgias: $2,1 \%$

6. Mareos: $1,1 \%$

7. Genitourinarios: $9,6 \%$

8. Gastrointestinales (GEA): 7,4\%

9. Cefaleas: $6,4 \%$

10. Ansiedad: $2,1 \%$

11. Picaduras: $3,2 \%$

12. Oftalmológicos: 7,4\%

13. Solicitud de información: $2,1 \%$

14. Respiratorios: $3,2 \%$

15. Heridas: $3,2 \%$
16. Dermatológicos: $3,2 \%$

17. Otorrinolaringológicos: $2,1 \%$

Y el destino tras la asistencia es:

- Su domicilio: $94,7 \%$

- Su domicilio / consultas externas: $3,2 \%$

- Ingreso: $2,1 \%$

Como vemos se trata en su mayoría de patología banal que podría fácilmente ser tratada en los centros de atención primaria, lo que nos lleva a indagar sobre la causa de elegir este centro para solicitar la asistencia, sobre todo en la población africana cuyas pautas socio-culturales se alejan de las nuestras más, con respecto a los otros dos grupos étnicos, de las nuestras y se precisa de un mayor grado de mediación cultural.

Así para este grupo, la distancia desde los núcleos habituales de residencia a los centros de Atención Primaria (A.P.) es significativamente mayor a la que recorren para acceder al hospital (Tabla 5), sin embargo, ello no supone un impedimento para acudir aquí a pesar de dejar atrás los otros centros. De hecho acuden a través de vehículo normalmente de algún conocido o allegado y es más, es práctica habitual el acudir en más de una ocasión en días sucesivos si la clínica del motivo de consulta no mejora de forma inminente si bien es verdad que las horas de máxima afluencia de población inmigrada no se diferencia de las de población general. Esto nos induce al análisis de las razones que argumentan para justificar su acceso directamente a la atención secundaria:

- En primer lugar indican que no conocen la disponibilidad de servicios de urgencias en atención primaria.

- En otras exponen la situación de que no pueden trabajar o no tienen quien los cuide y que precisan de ingreso hospitalario.

- Percepción de mayor accesibilidad horaria al hospital mientras que los centros de primaria, salvo el centro de urgencias sólo están abiertos en turno de mañana.

- Imposibilidad de interrumpir la jornada de trabajo para acudir al médico.

En definitiva, el perfil del inmigrante africano que acude al servicio de urgencias es la del varón joven que acude por patología generalmente banal; que no supone una interrupción de su actividad 
laboral, y cuya interrupción percibe como una amenaza por lo que demanda asistencia y recuperación rápida. Otra estampa típica es de un proceso fisiopatológico (traumatismo, deshidratación, malestar general, ...) que le impide acudir a las explotaciones agropecuarias pero no puede mantener tal situación puesto que no cuenta con reservas para su sustento o necesita alguien que le ofrezca unos cuidados mínimos que busca a través del ingreso. En este sentido debemos recordar que para la cultura árabe y musulmana los cuidados son dispensados por el género femenino, de tal manera que no es habitual que un varón mayor de edad se dedique al cuidado de otro y cubra las necesidades más básicas como la higiene, alimentación o acicalamiento por lo que a medida que el enfermo se va haciendo más dependiente queda más desprotegido y muchas veces es llevado al hospital más por necesidad de cuidados que de tratamiento médico.

En este sentido se plantea la necesidad de intervención desde el primer contacto con este grupo de población inmigrada que, por el conglomerado de características que presenta, vistas desde distintas perspectivas, se beneficiaría en un mayor grado de la mediación intercultural. Así desde el propio servicio de urgencias en la consulta de Recepción, Acogida y Clasificación de pacientes debe iniciarse un proceso de mediación que comprende actuaciones como Intermediación Cultural (Intervención NIC 7330) así como Guías del Sistema Sanitario (7400) o Educación Sanitaria (5510) en el más amplio abanico de posibilidades. Éste debe ser el inicio de un complejo entramado interdisciplinario que tenga como fin común alcanzar el mayor grado de bienestar físico y psíquico e integración sociocultural del individuo en la sociedad de recepción, siempre desde el respeto de las pautas culturales propias sin pretender una aculturación como fin último.

\section{CONCLUSIONES}

La disparidad de situaciones y polarización de los movimientos migratorios ha hecho que hoy por hoy, a pesar de reconocer este acontecimiento como global en nuestro entorno dé lugar a situaciones localmente diferenciadas que precisan de un análisis específico, como es el caso que tratamos. En estos casos la salud cobra un valor primordial en tanto que nos referimos a personas que buscan unas perspectivas de futuro que pueden verse interrumpidas por la falta de esta, y cuya pérdida es vivida especialmente como una amenaza. Por otro lado, las pautas culturales y realidad social a que se enfrentan una vez llegados no se corresponden con las ideales o expectativas que esperaban, no obstante siempre hay un afán de mejora y de prosperar. Por otro lado, se ven envueltos en la mayoría de las ocasiones en una sociedad receptora en la que no faltan prejuicios y estereotipos, con inexistencia de espacios de relación con la población autóctona y reticencias a la hora de alquilarles viviendas o aceptarlos por vecinos, lo que alimenta aún más los aspectos mencionados. Ante esta realidad de nuestra sociedad cambiante, la profesión enfermera debe ir evolucionando al ritmo que marcan los tiempos y adaptándose a las demandas que solicita nuestro centro de atención: el hombre como ser completo. Se justifica ahora más que nunca la visión holística del individuo pero siempre desde el estudio y conocimiento profundo de todas las perspectivas que lo definen. Además como elemento generador de salud y bienestar el profesional enfermero debe potenciar, más si cabe, su capacidad de interaccionar y trabajar en colaboración con otros profesionales no sólo del equipo sanitario sino que también de otras disciplinas que incluso desde otras administraciones pueden colaborar en lo que en definitiva podríamos denominar como salud comunitaria. En este aspecto la enfermería de atención especializada, a la que siempre se la ha relacionado con la más pura concepción biologicista del sistema sanitario, al ser en la mayoría de los casos el primer punto de relación entre el inmigrado y sistema sanitario, cobra importancia en tanto que depende en gran medida de su actitud y aptitud la posibilidad de que ese relación se forje y a partir de ella se inicie un verdadero proceso de integración en el que sea verdaderamente el individuo, con independencia de su lugar de procedencia, el eje de la atención sanitaria.

\section{BIBLIOGRAFÍA}

- AEED (1995) "Ante la marginación social las enfermeras responden", Libro de actas de las XVIII sesiones de trabajo organizadas por la Asociación Española de Enfermería Docente, Madrid. 
- Aguirre Raya D.(2004)"Fundamentos de la relación enfermera-persona sana $\mathrm{o}$ enferma" Rev Cubana Salud Pública ;30(4)

- Allué, M. "La gestión del morir: hacia una antropología del morir y de la enfermedad terminal" Jano 653.

- Asociación Pro derechos humanos de Andalucía.(2005)"Sobre la situación de las cortijadas y sus habitantes en el Campo de Níjar". Delegación de Almería. Edición propia.

- Bacote-Campinha J. (1996) A culturally competent model of nursing management. Surgical Services Management; 2 (5): 22-25.

- Baraza Cano, M P.(2005) En "Identificación de Diagnósticos Enfermeros en población inmigrante del Distrito Poniente de Almería”.Enf. Comun.; 1 (1)

- Barroso RZ, Torres EM.(2001) Fuentes teóricas de la enfermería profesional. Su influencia en la atención al hombre como ser biopsicosocial. Rev Cubana Salud Pública;27(1):118.

- Boff L.(1999) Saber Cuidar. Ética pelo humano- compaixão pela terra. $3^{\mathrm{a}}$ ed. Petrópolis. Editora Vozes.

- Brink, P.J. (1999) Transcultural versus cross-cultural. Journal of Transcultural Nursing.; 10(1), 6.

- Bushy A. (1992) Cultural considerations for primary health care: where do self-care and folk medicine fit? Holistic Nurs Pract.; 6 (3): 10-18.

- Colliére, F.(1993) "Promover la vida". De la práctica de las mujeres cuidadoras a los cuidados de enfermería. Ed. Interamericana, Madrid.

- Comelles, J.M.; Martínez A. (1993) en Enfermedad, Cultura y Sociedad, Ed. Universidad Complutense de Madrid, Madrid.

- "Cultura de los cuidados" Revista de Enfermería y Humanidades, seminario de Historia y Antropología de los cuidados enfermeros, Departamento de Enfermería, Universidad de Alicante.

- Ferro, N.(1991) en El instinto maternal o la necesidad de un mito, Ed. Siglo XXI de España editores, Madrid.

- García García, J. L. (1985) "Enfermedad y cultura", Jano. No 643-H, Madrid: 83-90.

- Gómez García, P.(2000) “Globalización cultural, identidad y sentido de la vida", Gazeta de Antropología, n 16, texto 1602

- Good, B. J.(2003) Medicina, racionalidad y experiencia. Una perspectiva antropológica. Bellaterra: Barcelona.

- Grossman D.(1994) Enhancing your 'cultural competence'. American Journal of Nursing. 58-62.

- Leininger, Madeleine (1970) Nursing and anthropology: Two worlds to blends. Ed. John Wiley \& Song. New York. EE.UU. - Leininger, Madeleine (1974) Health care dimension. Ed. F.A. Davis. Philadelphia. EE.UU.

- Malgesini, G. (y C. Giménez) (2000) Guía de conceptos sobre migraciones, racismo e interculturalidad. Madrid, Catarata.

- Marriner - Tomey, Ann, Raile Alligow, Martha (1999) Modelos y Teorías en Enfermería. Madrid: Editorial Harcourt Brace.

- Martínez, A. et al, (1998) en "Trabajo Social y Salud". Antropología de la medicina, $\mathrm{n}^{\circ} 29$, marzo, 1998 y n 31, diciembre, 1998.

- McKennis A.(1999) Caring for the islamic patient. Association of Operating Room Nurses. AORN Journa; 69 (6): 1.185-1.202.
- Perdiguero, E; Comelles, J. M. (eds.) (2000) Medicina y cultura. Estudios entre la antropología y la medicina. Bellaterra: Barcelona.

- Purnell L, Paulanka B, editores. (1998) Transcultural Health Care. Philadelphia: F. A. Company.

- Rodríguez Regueira, J. L. (2001) "Multiculturalismo. El reconocimiento de la diferencia como mecanismo de marginación social", Gazeta de Antropología, no 17, texto 17-04.

- Rojo N, García R. (2000) Sociología y salud. Reflexiones para la acción. Rev Cubana Salud Pública; 24(2):91-100.

- Siles J, editor. (2000) Antropología narrativa de los cuidados. Alicante: CECOVA.

- Solas, O; Ugalde, A. (1997) Inmigración: salud y políticas sociales. Escuela Andaluza de Salud Pública: Granada.

- Vasquez Trussi, Marta (2001) El cuidado de enfermería desde la perspectiva transcultural: una necesidad en un mundo cambiante. http://tone.udea.edu.co/revista/

\section{ANEXO:}

Tabla 1. - Evolución cifras absolutas anuales de Inmigrantes empadronados en España.

\begin{tabular}{|r|r|r|r|}
\cline { 2 - 4 } \multicolumn{1}{c|}{} & $\begin{array}{c}\text { Ambos } \\
\text { sexos }\end{array}$ & \multicolumn{1}{c|}{ Varones } & Mujeres \\
\hline $\mathbf{1 9 9 8}$ & 56.264 & 28.103 & 28.161 \\
\hline $\mathbf{1 9 9 9}$ & 97.983 & 49.495 & 48.488 \\
\hline $\mathbf{2 0 0 0}$ & 329.394 & 177.279 & 152.11 .5 \\
\hline $\mathbf{2 0 0 1}$ & 392.615 & 209.852 & 182.763 \\
\hline $\mathbf{2 0 0 2}$ & 441.477 & 231.893 & 209.584 \\
\hline $\mathbf{2 0 0 3}$ & 428.008 & 222.306 & 205.702 \\
\hline $\mathbf{2 0 0 4}$ & 642.617 & 353.122 & 289.495 \\
\hline $\mathbf{2 0 0 5}$ & 4.391 .484 & 2.296 .052 & 2.095 .432 \\
\hline
\end{tabular}

Fuente: Instituto Nacional de Estadística (INE).

Tabla 2.- Distribución de la población inmigrante por grupos de edad y sexo. Año 2005.

\begin{tabular}{|c|c|c|c|c|}
\hline & \multirow{2}{*}{$\begin{array}{l}\text { Varones } \\
\text { Valor } \\
\text { ahsolute }\end{array}$} & \multirow{2}{*}{$\begin{array}{c}\text { Mujeres } \\
\text { Valor } \\
\text { aboolute }\end{array}$} & \multicolumn{2}{|c|}{ Anbes sexas } \\
\hline & & & $\begin{array}{c}\text { Valor } \\
\text { ahsolute }\end{array}$ & Porrebiaje \\
\hline De 0 a 4 & 86434 & 81.518 & 167952 & 4,50 \\
\hline De 5 a 9 & 93549 & 809.499 & 182.039 & 4.88 \\
\hline $\begin{array}{c}\text { De } 10 \text { a } \\
14\end{array}$ & 92173 & 86549 & 178722 & 4,79 \\
\hline $\begin{array}{c}\text { De } 15 \text { a } \\
19\end{array}$ & W9.399 & 91.0015 & 191.304 & 5,13 \\
\hline $\begin{array}{c}D e 20 a \\
24\end{array}$ & 193038 & 182.322 & 375360 & 10,06 \\
\hline $\begin{array}{c}\mathrm{D} e \\
29\end{array}$ & 318440 & 263.137 & $881.5 \pi 7$ & 15,59 \\
\hline $\begin{array}{c}D e 30 \text { a } \\
34\end{array}$ & 309363 & 255.2001 & 544.563 & 14,60 \\
\hline $\begin{array}{c}\mathrm{De} 35 \mathrm{a} \\
39\end{array}$ & 246154 & 188.775 & 434029 & 11,66 \\
\hline $\begin{array}{c}D e 40 a \\
44\end{array}$ & 174.966 & 143.571 & 318.537 & 8,54 \\
\hline
\end{tabular}




\begin{tabular}{|c|c|c|c|c|}
\hline$\underbrace{D e}_{49} 45$ a & 117.646 & $1(13.749)$ & 221.395 & 5,93 \\
\hline $\begin{array}{c}\text { De } 59 a \\
54\end{array}$ & 73.961 & 75.571 & 149.532 & 4,01 \\
\hline $\begin{array}{c}\text { De } 55 \text { a } \\
59\end{array}$ & 52355 & 90.229 & 111.84 & 2,99 \\
\hline $\begin{array}{c}\text { De } 60 \text { a } \\
64\end{array}$ & 45683 & 48.654 & 93.781 & 2,51 \\
\hline $\begin{array}{c}\text { De } 65 \\
69\end{array}$ & 38024 & 36.413 & 74.437 & 2,00 \\
\hline $\begin{array}{c}\text { De } 70 \text { a } \\
74\end{array}$ & 24.432 & 23.304 & 47.736 & 1,28 \\
\hline $\begin{array}{c}\text { De } 75 \text { a } \\
79\end{array}$ & 14.593 & $14 \times 62$ & 29,455 & 0,79 \\
\hline $\begin{array}{c}\text { De } 80 \text { a } \\
84\end{array}$ & 8018 & 0,381 & 17,000 & 0,46 \\
\hline Mas de 85 & 4.411 & 6.197 & 10008 & 0,28 \\
\hline TOYTAL & $1.902 \times 134$ & $1.73 \times .576$ & 3.730610 & 100,00 \\
\hline
\end{tabular}

Fuente: Instituto Nacional de Estadística (INE).

Tabla 3.- Distribución población inmigrante por Provincias en Andalucía.

\begin{tabular}{|c|c|c|}
\hline & $\begin{array}{l}\text { Valot } \\
\text { Abs. }\end{array}$ & क्ष: \\
\hline ANDALUCIA & 88804 & 100 \\
\hline Alaneria & 25.2085 & 29,48 \\
\hline Cudiz & 5310 & 5.97 \\
\hline Cóndoba & 3.433 & 3,86 \\
\hline Granada & 8298 & 9,33 \\
\hline Hoelva & 4.283 & 4,82 \\
\hline Jake & 2.194 & 2,47 \\
\hline Málaga & 31.523 & 35,46 \\
\hline Sevilla & $764 \mathrm{~B}$ & 8,60 \\
\hline
\end{tabular}

Fuente: Instituto Nacional de Estadística (INE).
Tabla 4.- Proporción de inmigrantes por autóctonos en Provincias de Andalucía.

Fuente: Instituto Nacional de Estadística (INE).

\begin{tabular}{|c|c|c|c|}
\hline & INMICRANTES & $\begin{array}{l}\text { POBL } \\
\text { TOTAL }\end{array}$ & $\tau$ \\
\hline Nmeria & 26205 & 612315 & 4.28 \\
\hline Cádix & 5310 & 1.180817 & 0.04 \\
\hline Cóndeba & 3.433 & 784376 & 0,44 \\
\hline Granada & 8298 & R60 BSG & 0.96 \\
\hline Heelva & 4283 & 483.792 & 0.89 \\
\hline Jaén & 2194 & 660284 & 0,33 \\
\hline Málaga & 31.523 & 1.453 .409 & 2,17 \\
\hline Sevilla & 7.648 & $1.813 .90 \mathrm{~K}$ & 0.42 \\
\hline ANDALUCIA & \$8.894 & 7.899 .799 & 1.13 \\
\hline
\end{tabular}

Tabla 5.- Distancia (en Km) desde los principales núcleos de viviendas de población magrebí y sudsahariana a los centros de Atención Primaria (A.P.) y Hospital de Alta Resolución El Toyo.

\begin{tabular}{|c|c|c|c|}
\hline (वит) & 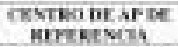 & $\begin{array}{c}\text { kuckinos } \\
\text { re Af }\end{array}$ & $\begin{array}{c}k \mu \mathrm{Hn} \\
\mathrm{Tan}\end{array}$ \\
\hline IIMSTA? & cavgrite aycen & $\ln _{2}$ & 21 \\
\hline insoutage & Caviquiciokons & $\overline{7}$ & 3 \\
\hline 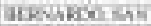 & caveicakes & 9 & 34 \\
\hline 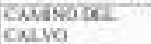 & 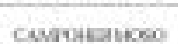 & 23 & 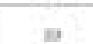 \\
\hline 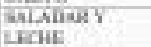 & Cunprycaskicen & $a$ & $x$ \\
\hline बतs & swisiteo & 25 & 25 \\
\hline Eavaring: & INvisicket & ; & $\sin$ \\
\hline 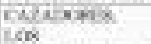 & AMNISIEQT & is & $n$ \\
\hline Fen thoula: & A. vivitewi & 7 & 4 \\
\hline Fonurane. & Aviniteos & 29 & 175 \\
\hline $\begin{array}{l}\text { intox, cunpo } \\
\text { ger. }\end{array}$ & savintrent & at & 34 \\
\hline 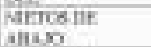 & ravisicho & 18 & $m$ \\
\hline $\cos 20$ & 3aviliteno & I & n \\
\hline
\end{tabular}

Elaboración propia.

Graffico 1.- Distribueión de la población inmigrada por Comunidades Autonomas.

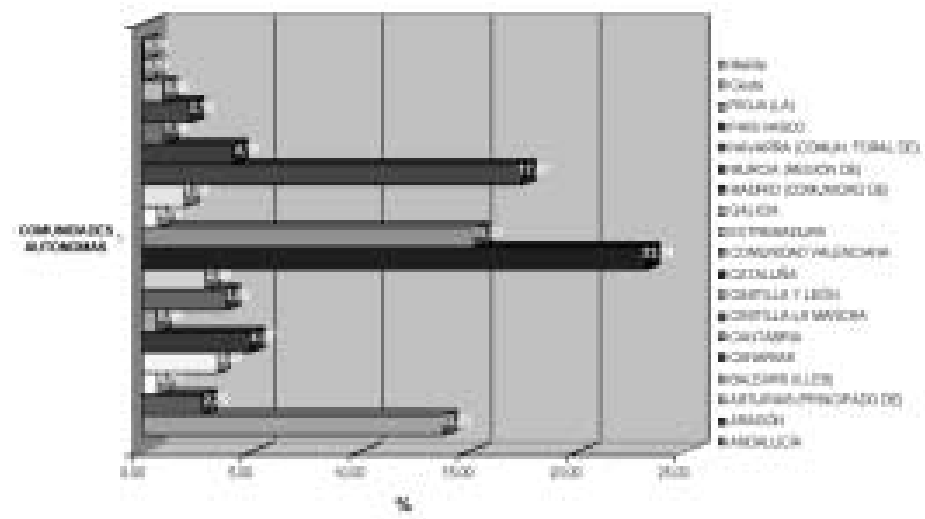

